\title{
EXPERIMENTAL RESEARCH AND CFD CALCULATIONS BASED INVESTIGATIONS INTO GAS FLOW IN A SHORT SEGMENT OF A HEAVILY WORN STRAIGHT THROUGH LABYRINTH SEAL
}

\author{
Damian Joachimiak \\ Piotr Krzyślak \\ Poznań University of Technology, Poland
}

\begin{abstract}
Steam turbines are used as propulsion components in not only power plants but also on merchant and naval ships. The geometry of the steam turbine seals changes throughout the machine life cycle. The rate of deterioration of these seals, in turn, affects heavily the efficiency of the thermal machine. However, the literature overview does not provide any research reports on flow phenomena occurring in heavily deteriorated seals. The paper describes the course and results of investigations into a model straight through labyrinth seal composed of 4 discs, each with the slot height of $2 \mathrm{~mm}$. The investigations have been conducted with air as the working medium. Changes of gas flow parameters due to wear were analysed. Based on the experimental data, more intensive leakage was observed as the result of the increased slot height. The static pressure distribution along the examined segment was measured. The experimentally recorded distribution differed remarkably from the theoretical assumptions. Another part of the experimental research focused on comparing the gas velocities at points situated upstream of the first and second seal disc. The velocity measurements were carried out using a constant temperature wire probe. This part of the investigations provided opportunities for analysing the influence of seal wear on gas flow conditions in the seal segment. The paper compares the results of the experimental research with those obtained using the CFX software. The presented results of velocity distributions provide a clear picture of the nature of the gas flow in the seal, which enables its analysis.
\end{abstract}

Keywords: labyrinth seals, turbomachines, fluid-flow machines, experiment, CFD, gas velocity

\section{INTRODUCTION}

Steam turbines are used for propulsion of marine vessels and power generators in power plants. The initial average gap height in the $13 \mathrm{~K} 215$ turbine glands amounts to $0.8 \mathrm{~mm}$. When the service life of the turbine comes to an end (overhaul interval) and the turbine is dismantled, the average height of the gap increases to $1.95 \mathrm{~mm}$ [17], as a result of significant wear of seals taking place during turbine operation between overhauls. This effect has a great influence on the thermal turbine efficiency [8]. Ref. [7] presents a labyrinth seal diagnosing method which consists in distorting the steam flow in the seal. This distortion is achieved through releasing a known amount of steam in a given cross-section of the seal. The pressure values measured upstream of the seal in the extraction plane and downstream of the seal, in combination with the known amount of steam mass flow released through the extraction, make the basis for estimating the amount of leakage in the turbine gland. The present paper treats on the experimental research and CFD calculations of a model segment of a straight through labyrinth seal. In the performed tests, air was used as the working medium. The examined type of seal is used in turbines as external and shroud seals, as well as base seals of stator blade rings in medium and low-pressure steam turbine stages located far from thrust bearings [14]. These components are responsible for reducing both the stage leakages, and the external turbine leakages.

The abovementioned wear of seals is a source of increased leakage flow and decreased main flow, with the turbine efficiency loss as a further consequence. The influence of the one-sided shroud seal composed of two disks on the flow in axial low-speed turbine passages has been analysed in $[2,3]$. Among other issues, these works examined experimentally the influence of the leakages on the mixing loss and secondary flows. A segment of a four-slot one-sided seal was the subject of the research described in [5]. The experimental research and numerical calculations performed in this work were related to the energy loss due to flow turbulence, described using the friction loss. 
The investigations into dynamic pressure and gas velocity distributions in a chamber of a double-sided seal have been presented in [1]. The results of experimental research were compared with those obtained from numerical calculations.

In $[10,11]$, the numerical and experimental research of the gas flow in a shroud clearance of a turbine stage have been presented. The analysis pertained to pressure distributions in one-sided and two-sided seals with different disc numbers and geometries.

The gas flow in the shroud clearance of the rotor stage of a high-pressure turbine has been studied in [15], based on CFD calculations performed for the straight through labyrinth seal. In [13], the leakage of a 6-disc segment of a one-sided seal was compared with that of the 5-disc stepped seal, based on the CFD calculations and the one-dimensional theory. The experimental research oriented on determining friction loss coefficients for short segments of a straight through labyrinth seal has been presented in $[12,18]$. The influence of the shaft speed on the leakage of the double-sided seal segment has been shown in [9]. The results of the experimental research and CFD calculations performed on a segment composed of 20 discs have been compared in [6]. The leakage and the static pressure distribution in the segment were analysed based on the experiment and calculations performed using the CFX software. All the above works pertain to nominal slot heights, while, thus far, no research has been reported on deteriorated seals. This paper presents the results of experimental research for a one-sided seal with the gap height of $2 \mathrm{~mm}$, the geometry of which is similar to that of the interstage seal of a steam turbine.

\section{RESEARCH STAND}

The experimental research was performed on the test stand [7] composed of the compressor, tank, mass flow measurement system, and the casing (Fig. 1). The geometry of the examined model straight through labyrinth seal is shown in Fig. 2, along with the locations of pressure measurement points.

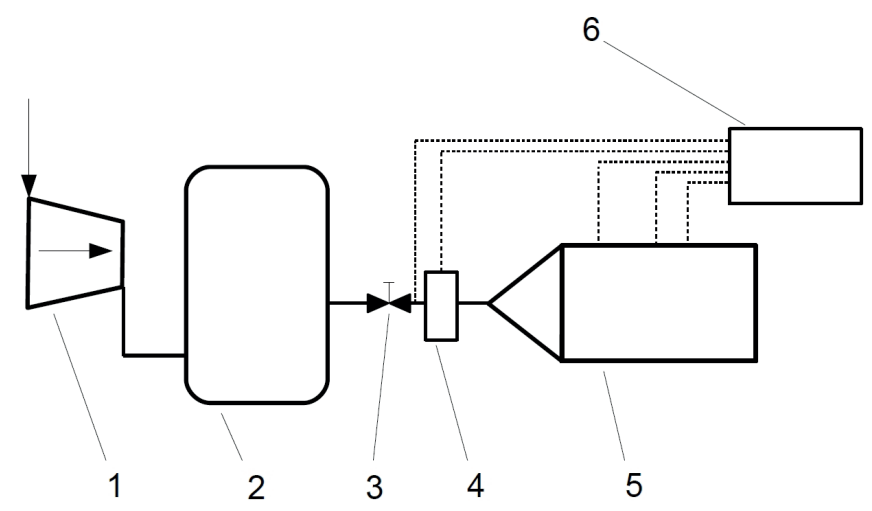

Fig. 1. Schematic of the labyrinth seal test stand: 1 - compressor, 2 - main tank, 3 - regulator valve, 3 - inlet channel, 4 - orifice, 5 - model labyrinth seal segment, 6 - measurement system
In the experimental research, absolute pressure converters with the measurement range of $0-5 \cdot 10^{5} \mathrm{~Pa}$ and the measurement accuracy of $\pm 0.25 \%$. were used. Moreover, pressure difference converters with the measurement range of $0-025 \cdot 10^{5} \mathrm{~Pa}$ and the measurement accuracy of $\pm 0.2 \%$ were applied. The temperature was measured using T-thermocouples. The mass flow was measured using an orifice with pressure measurement at the disc. The average height of the gap of the examined segment was $2 \mathrm{~mm}$.

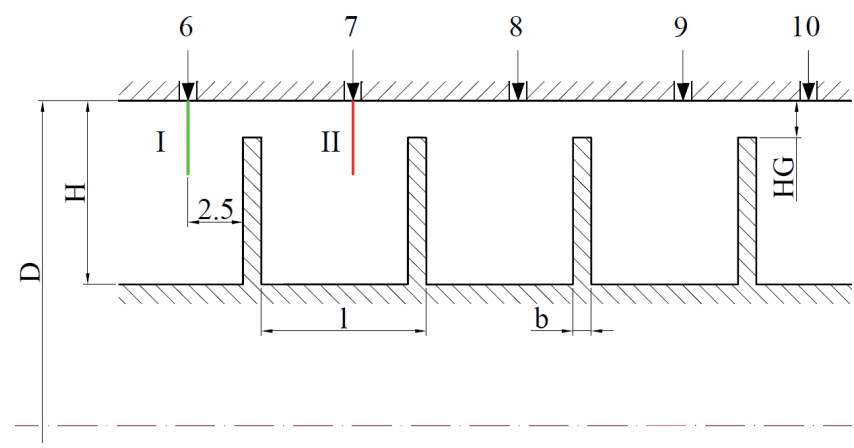

Fig. 2. Geometry of the examined seal segment with measurement planes I and II, and pressure measurement points 6-10

Tab. 1. Geometry dimensions

\begin{tabular}{|c|c|l|}
\hline Mark & Quantity & \multicolumn{1}{|c|}{ Size } \\
\hline $\mathrm{D}$ & Seal diameter & $150 \mathrm{~mm}$ \\
\hline 1 & Scale length & $10 \mathrm{~mm}$ \\
\hline $\mathrm{H}$ & Seal segment height & $10 \mathrm{~mm}$ \\
\hline HG & Gap height & $0.3,0.5,0.7,1,1.5,2 \mathrm{~mm}$ \\
\hline $\mathrm{b}$ & Disc thickness & $1 \mathrm{~mm}$ \\
\hline
\end{tabular}

The measurement planes I and II (Fig. 2) were located as close to the slot as possible, in order to obtain the most accurate information on the velocity of the gas reaching the slot. At the same time, each location was sufficiently far from the disc to avoid the area where the axial gas velocity component is dominant. The locations of the measurement planes were selected based on preliminary CFD simulations (Fig. 3).

The velocity field was measured using a constant temperature probe in two planes of $4 \mathrm{~mm}$ in height (Fig. 2, 3). One of these planes was situated at $2.5 \mathrm{~mm}$ upstream of the first disc, and the second one at the same distance upstream of the second disc.

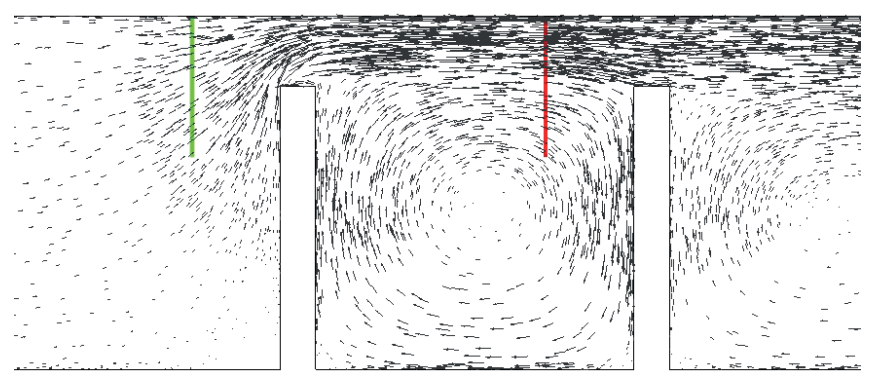

Fig. 3. Flow simulation upstream of the first and second disc of the labyrinth seal (velocity vectors) for $\dot{m}=0.5 \mathrm{~kg} / \mathrm{s}, H G=2 \mathrm{~mm}$ 
The gas velocity measurement in the first plane provided the information that the air velocity field was undisturbed, which resulted from the continuity of the flow. The velocity distribution in the second plane contains the information that the gas velocity can be relatively high, compared to that observed in the first plane. This velocity depends on the kinetic energy dissipation rate in the first chamber, the pressure decrease in the seal, and the seal geometry.

\section{CFD ANALYSIS}

The investigations refer to the segment in which the gas flow is assumed axisymmetric. Geometry discretization was performed in the CFX Mesh software. Wall boundary layers were assumed on the body surface, on lower parts of the chambers, and on the discs.

The examined geometry was described by the mesh with 138999 nodes and 297901 elements. The mesh was refined in the gap area, in which greatest gas pressure and velocity changes were expected (Fig. 4). The $\mathrm{y}^{+}$parameter was equal to 0.01 in the disc edge area, and to 0.07 on the body surface around the slot.

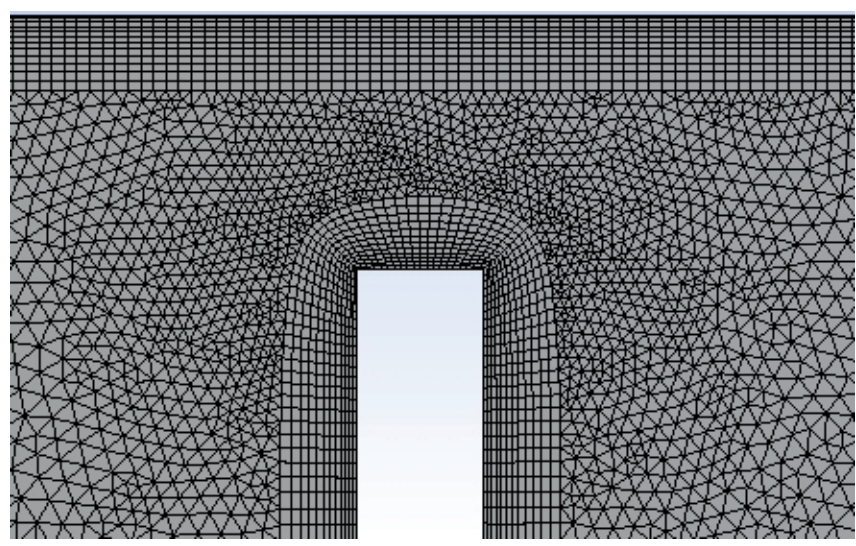

Fig. 4. Mesh used for flow calculations around the slot with $H G=2 \mathrm{~mm}$

In the inlet plane to the computational domain of the examined seal segment, the mass flow and the normal flow direction were set, while the average static pressure was set in the outlet plane. The values of these parameters which were used in the numerical calculations were obtained from the experiment. The calculations were made for the air as compressible gas.

The paper presents the results of CFX (RANS) calculations, which consisted in solving momentum, continuity, energy, and turbulence equations [20]. The stationary calculations were made with the false time step equal to $1 \cdot 10^{-5} \mathrm{~s}$. The gas flow in the examined geometry was generated by a significant pressure gradient. The gas velocity was high in the slot areas, and much lower in the chambers. Out of the available turbulence models in the CFX software, the SSG model was applied. This model provides good solutions for boundary layers, and recirculating and high curvature flows.

\section{RESULTS OF EXPERIMENTAL RESEARCH}

The mass flow through the seal depends on the pressure decrease. The mass flow measurement was performed for segments with gap heights $\mathrm{HG}=0.3,0.5,0.7,1,1.5$ and $2 \mathrm{~mm}$ (Fig.1, Tab.1). The results are presented as pressure ratios $\mathrm{p}_{1} /$ $\mathrm{p}_{2}$. Figure 5 reveals that the mass flow increase due to wear is $\mathrm{m} / \mathrm{m}_{\mathrm{HF}=0.3}=1.6$, starting from $\mathrm{HG}=0.3$ to $0.5 \mathrm{~mm}$ for the pressure of $\mathrm{p}_{1} / \mathrm{p}_{2}=2$, while for the wear from $\mathrm{HG}=0.3$ to 0.7 the leakage increase is $\mathrm{m} / \mathrm{m}_{\mathrm{HG}=0.3}=2.25$. The mass flow grows rapidly when the pressure decreases for segments with gap heights HG 1,1.5 and $2 \mathrm{~mm}$.

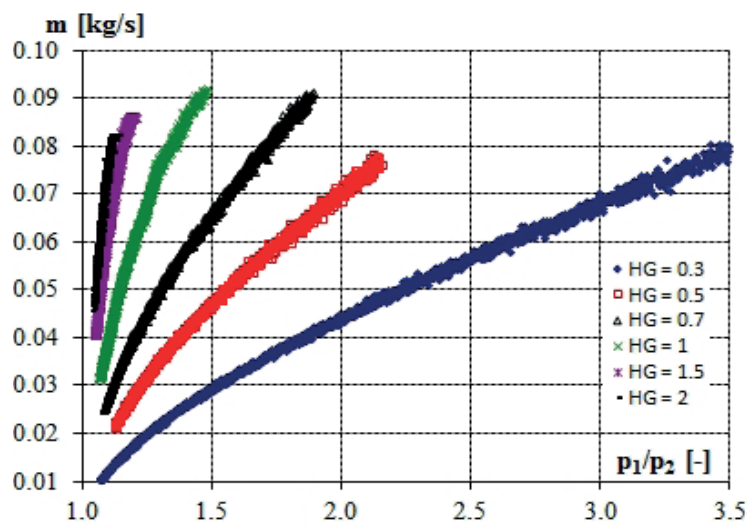

Fig. 5. Mass flow through the seal with geometry shown in Fig. 4 and gap height equal to $H G=0.3,0.5,0.7,1,1.5$ and $2 \mathrm{~mm}$. The scale of $10 \mathrm{~mm}$ is a function of pressure decrease

The data shown in Fig. 5 testify that the leakage through the segment of the straight through labyrinth seal drastically increases as a result of wear.

The range of the experimentally analysed seal pressure ratio was limited by high gas velocities recorded by a fibre probe in plane II. This probe has the velocity measurement range limited to $110 \mathrm{~m} / \mathrm{s}$.

Figure 6 presents static pressure distributions along the seal segment with gap height $\mathrm{HG}=2 \mathrm{~mm}$, obtained in the experiment and CFX simulations for the mass flow $\dot{m}=0.05$ $\mathrm{kg} / \mathrm{s}$.

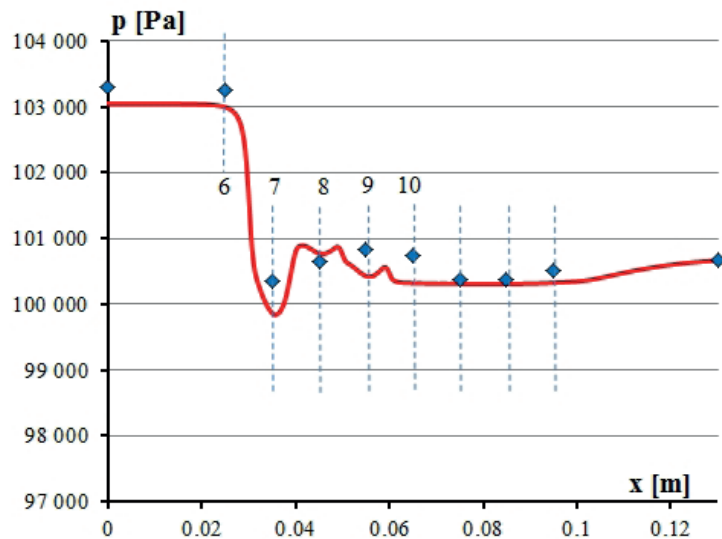

Fig. 6. Static pressure distributions in the seal segment, for mass flow $\dot{m}=0.05$ $\mathrm{kg} / \mathrm{s}$ and $H G=2 \mathrm{~mm}$ : points - data from experiment, $6-10$ (marks of points as per Fig. 2), continuous line - CFX data 
The experimentally recorded pressure distribution (blue points) is similar to that obtained in the CFX simulations (red curve in Fig. 6). The relative error of the resultant static pressure in the inlet plane obtained in the CFX simulations did not exceed $2 \%$, compared to the pressure recorded in the experiment for the examined cases.

According to the data published in $[6,7,16,19]$, the theoretical pressure distribution in the seal with small slot height decreases evenly and the gas velocity in the slots increases gradually.

The static pressure distribution obtained for the examined straight through labyrinth seal significantly differs from those observed for segments with smaller gap heights - HG = $0.2-1 \mathrm{~mm}[5,6]$.

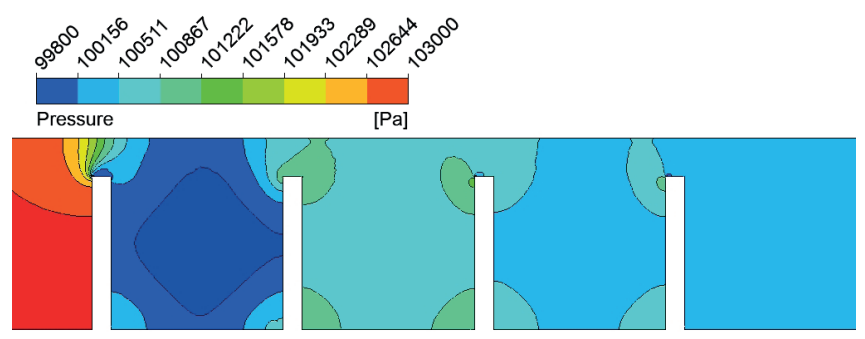

Fig. 7. Static pressure distribution of the working gas obtained in CFX simulations for $\dot{m}=0.05 \mathrm{~kg} / \mathrm{s}$ and $H G=2 \mathrm{~mm}$

The presented measurement data and the CFX simulations (Figs. 6 and 7) testify that the greatest pressure decrease in the segment occurs in the first slot area, while the decompression does not take place in the further part of the segment. The lowest pressure throughout the segment, observed directly behind the first slot in the chamber, is just the result of significant gas decompression. The value of this pressure affects the air velocity profile in the measurement plane II. Figure 8 presents the velocity distributions in the examined segment.

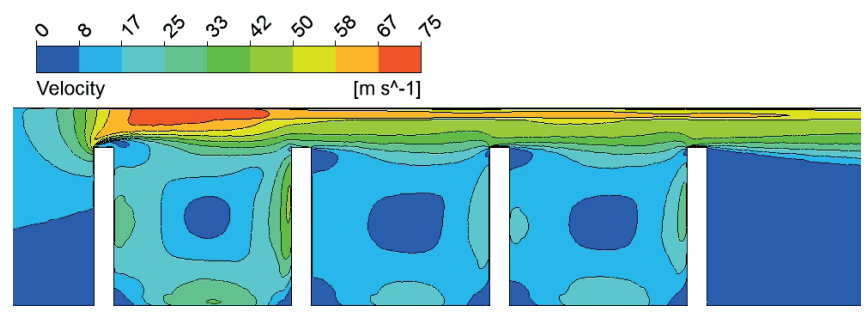

Fig. 8. Gas velocity distribution obtained in CFX simulation for the mass flow $\dot{m}=0.05 \mathrm{~kg} / \mathrm{s}$ and $H G=2 \mathrm{~mm}$

The velocity of the gas reaching the second slot is approximately equal to $75 \mathrm{~m} / \mathrm{s}$. In the upper part of the chambers of the examined slot, high and not decreasing gas flow velocities can be observed (Fig. 8).

\section{VELOCITY DISTRIBUTIONS OBTAINED FROM MEASUREMENT AND CFX SIMULATION}

The velocity vector distributions in planes I and II were measured using a constant temperature single fibre probe, which enabled point velocity measurements. Figure 9 presents the velocity profiles obtained from the experiment and from CFX simulations for three different mass flows.

a)
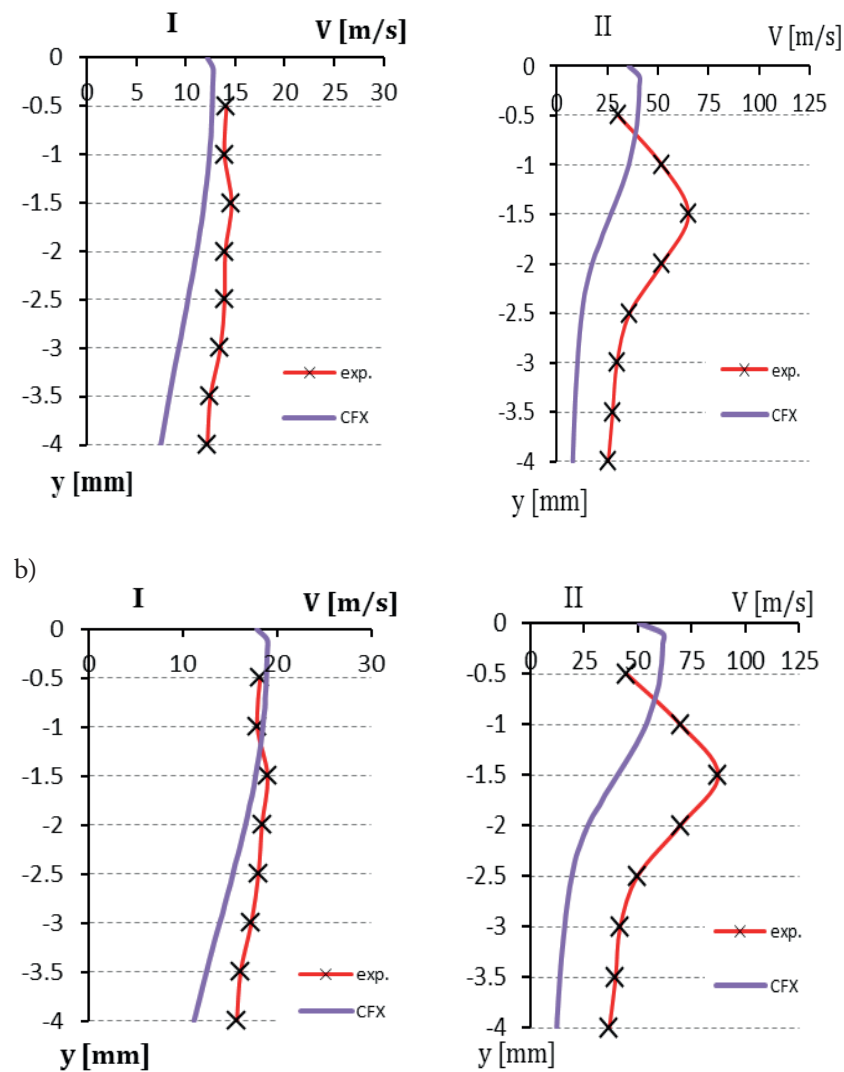

c)
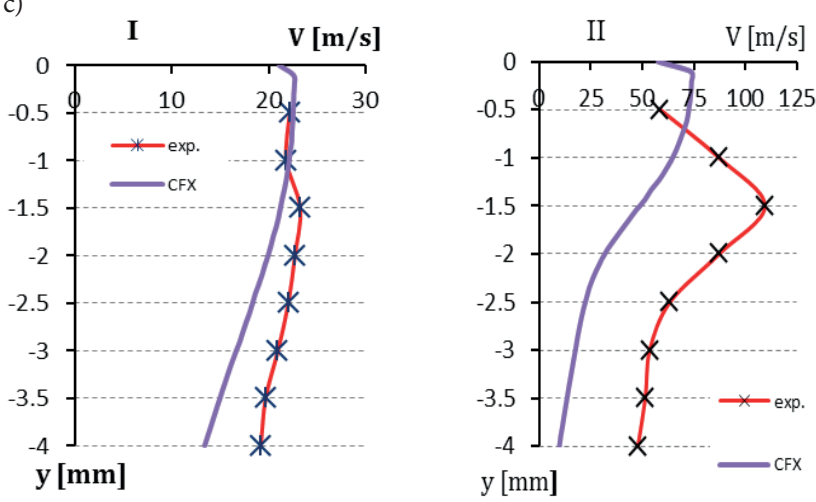

Fig. 9. Gas velocity vector distributions in seal segment with $H G=2 \mathrm{~mm}$, for mass flows: a) $0.03 \mathrm{~kg} / \mathrm{s}, b) 0.04 \mathrm{~kg} / \mathrm{s}, \mathrm{c}$ ) $0.05 \mathrm{~kg} / \mathrm{s}$ Red lines with points - measurement, continuous blue lines - CFX simulation.

In the first measurement plane, the velocity distributions obtained from the measurement and CFX simulation for 
the analysed mass flows are even and have similar values. The velocity profile shapes in the first measurement plane are the effect of the undisturbed flow upstream of the first disc and a significant height of the slot, compared to the height of the chamber. It results from the CFX data that the maximum gas velocity in the upper part of plane II is almost three times as high as that in plane $\mathrm{I}$, and its maximum values for the examined mass flows of $0.03,0.04,0.05 \mathrm{~kg} / \mathrm{s}$ are equal to 41,62 and $75 \mathrm{~m} / \mathrm{s}$ respectively. These maximum velocities are recorded near the wall of the body. The maximum gas velocities obtained in the experiment in plane II are almost four times as high as those in plane I. For the analysed mass flows, they are equal to 66,87 and $110 \mathrm{~m} / \mathrm{s}$ and are situated lower than in the CFX data, at a distance of $1.5 \mathrm{~mm}$ from the body wall.

The data shown in Figs. 7, 8 and 9 make the basis for a hypothesis that the velocity increase in plane I results from flow area reduction, while that recorded in plane II is the effect of small kinetic energy dissipation (Fig. 8, 9) and low static pressure in the first chamber (Fig. 6, 7).

In decompression measurements performed with the aid of constant temperature probes, air humidity change with respect to that in which the probe characteristic was determined is not allowed. The divergence between the velocity values recorded in the experiment and those obtained from CFX simulations may result from limited accuracy of probe location and from the presence of a hole through which the probe was introduced. The results obtained using the CFX software heavily depend on the applied turbulence model.

\section{CONCLUSIONS}

The presented results of seal segment investigations indicate that high wear of a seal significantly increases the leakage through it. This conclusion is confirmed by comparing velocity distributions in planes I and II, obtained from the measurements and CFD calculations. The results presented in the paper indicate high gas acceleration in the first slot area, with simultaneous slight velocity reduction in the first chamber. In seals with the gap to chamber height ratio, $\mathrm{HF} / \mathrm{H}$, equal to 0.2 , high gas flow velocities are observed over the disc edges. The recorded data clearly show the effect of kinetic energy carry-over of the gas in the segment.

This is the effect of gas decompression within the first slot. Both the calculations and the measurements testify that the static pressure decrease is small in the outstanding part of the seal.

For a heavily worn seal, the leakage of the working medium is mainly reduced by gas expansion in the first slot of the segment, while in the further part of the seal it is mainly generated by tangential tensions between the gas flow and the seal walls, and by kinetic energy dissipation in the chambers.

The presented static pressure distributions which were obtained in the experiment and CFD calculations for the worn seal illustrate its uneven course throughout the seal. The thus far applied Zalf, Stodola, Egli or Hodkinson models $[4,14]$ have revealed good accuracy for seals with nominal and slightly worn geometries. For heavily worn geometries, the mass flow values calculated using the abovementioned models need to be corrected.

The results presented in the paper testify that the leakage of the working medium in worn straight through labyrinth seals of steam turbines can grow in an uncontrolled way, the consequence of which may be significant drop in turbine efficiency.

\section{REFERENCES}

1. Alexander O. Pugachev a, Yury A. Ravikovich b, Leonid A. Savin., Flow structure in a short chamber of a labyrinth seal with a backward-facing step, Elsevier, Computers \& Fluids, 114, pp. 39-47, 2015

2. Anker J. E., Mayer J. F., Stetter H., Computational Study of the Flow in an Axial Turbine with Emphasis on the Interaction of Labyrinth Seal Leakage Flow and Main Flow, E. Krause et al. (eds.), High Performance Computing in Science and Engineering 2001, Springer-Verlag Berlin Heidelberg, 2002.

3. Anker J. E., Mayer J. F., Casey M., The impact of rotor labyrinth seal leakage flow on the loss generation in an axial turbine, Proceedings of the Institution of Mechanical Engineers, Vol. 219, Part A, Journal Power and Energy, 2005

4. Hodkinson B., Estimation of the leakage through a labyrinth gland, Proceedings of the institution of Mechanical Engineers 141, 1939

5. Hu D., Jia L., Yang L., Dimensional Analysis on Resistance Characteristics of Labyrinth Seals, Journal of Thermal Science, Vol.23, No.6, pp. 516-522, 2014

6. Joachimiak D., Krzyślak P., Comparison of results of experimental research with numerical calculations of a model one-sided seal, Archives of Thermodynamics, Vol. 36, No. 2, pp. 61-74, 2015

7. Joachimiak D.: The labyrinth seals research with extraction. PhD thesis, Poznan University of Technology, Poznan 2013 (in Polish).

8. Krzyślak P., Winowiecki M., A method of diagnosing labyrinth seals in fluid-flow machines, Polish Maritime Research 3(57), 15, pp. 38-41, 2008

9. Li J., Wen K., Wang S., Jiang S., Kong X., Experimental and numerical investigations on the leakage flow characteristics of labyrinth seals, Heat Mass Transfer and Energy Conversion AIP Conf. Proc. 1547, pp. 164-172, 2013 
10. Stępień R., Kosowski K., Remarks on aerodynamic forces in seals of turbine stages, Polish Maritime Research, Special issue 2009/S1; pp. 58-63

11. Stępień R., Kosowski K., Piwowarski M., Badur J.: Numerical and Experimental Investigations into Pressure Field in Blade Shroud Clearance, Part II: Numerical Analysis, Task Quarterly, 2003

12. Thiekle G., Stetter H.; Identification of friction factors for modelling the exciting forces caused by flow in labyrinth seals, Rotordynamics '92, Springer-Verlag London, 1992

13. Tong Seop Kim, and Kyu Sang Cha, Comparative analysis of the influence of labyrinth seal configuration on leakage behavior, Journal of Mechanical Science and Technology, pp. 2830-2838, 2009

14. Traupel W. Termische turbomaschinen, Ester B., Springer 2001

15. Wan-Fu Zhang a, Jian-Gang Yang b, Chun Li a, Yong-Wei Tian, Comparison of leakage performance and fluidinduced force of turbine tip labyrinth seal and a new kind of radial annular seal, Computers \& Fluids, 105, pp. 125-137, 2014

16. Trütnovsky K., Berührungsfreie Dichtungen, Grundlagen und Anwendungen der Strömung durch Spalte und Labyrinthe, VDI-Verlag bh Düsseldorf, Verlag des Vereins Deutscher Ingenieure 1964.

17. Winowiecki M., The method of diagnosing labyrinth seals flow machines. PhD thesis, Poznan University of Technology, Poznan 2009 (in Polish)

18. Yamada, Y., On the pressure loss of flow between rotating co-axial cylinders with rectangular grooves, Bulletin of the JSME, 5 (20), pp 642-651. 1962

19. Zimmerman H. and Wolff, K. H., 1987, “Comparison between Empirical and Numerical Labyrinth Flow Correlations," ASME 87-GT-86.

20. ANSYS. ANSYS CFX-solver theory guide. ANSYS, Inc., Release 14.5; 2012

\section{CONTACT WITH THE AUTHOR}

Damian Joachimiak

e-mail:damian.joachimiak@put.poznan.pl

Piotr Krzyślak

e-mail:piotr.krzyslak@put.poznan.pl

Poznań University of Technology

Piotrowo 3

60-965 Poznan

Poland 